\title{
Delta activity encodes taste information in the human brain
}

Raphael Wallroth ${ }^{1,2}$, Richard Höchenberger ${ }^{3}$, \& Kathrin Ohla ${ }^{1,2,3}$

1 Psychophysiology of Food Perception, German Institute of Human Nutrition PotsdamRehbruecke, 15448 Nuthetal, Germany

2 NutriAct - Competence Cluster Nutrition Research Berlin-Potsdam

3 Cognitive Neuroscience, Institute of Neuroscience and Medicine (INM-3), Research Center Jülich, 52428 Jülich, Germany

\section{Corresponding author:}

Kathrin Ohla, $\mathrm{PhD}$

Research Center Juelich

Institute of Neuroscience and Medicine (INM3)

Leo-Brandt-Str. 5

52428 Juelich, Germany

Email:k.ohla@fz-juelich.de 


\section{Abstract}

2 The categorization of food via sensing nutrients or toxins is crucial to the survival of any organism.

3 On ingestion, rapid responses within the gustatory system are required to identify the oral stimulus

4 to guide immediate behaviour (swallowing or expulsion). The way in which the human brain

5 accomplishes this task has so far remained unclear. Using multivariate analysis of 64-channel scalp

6 EEG recordings obtained from 16 volunteers during tasting salty, sweet, sour, or bitter solutions,

7 we found that activity in the delta-frequency range (1-4 Hz; delta power and phase) has information

8 about taste identity in the human brain, with discriminable response patterns at the single-trial level

9 within $130 \mathrm{~ms}$ of tasting. Importantly, the latencies of these response patterns predicted the point

10 in time at which participants indicated detection of a taste by pressing a button. Furthermore, taste

11 pattern discrimination was independent of motor-related activation and encoded taste identity

12 rather than other taste features such as intensity and valence. On comparison with our previous

13 findings from a delayed taste-discrimination task (Crouzet et al., 2015), taste-specific neural

14 representations emerged earlier during this speeded taste-detection task, suggesting a goal-

15 dependent flexibility in gustatory response coding. Together, these findings provide the first

16 evidence of a role of delta activity in taste-information coding in humans. Crucially, these neuronal

17 response patterns can be linked to the speed of simple gustatory perceptual decisions - a vital

18 performance index of nutrient sensing.

19

20 Keywords: gustation, taste quality, EEG, MVPA, delta 
23 The internal representation of sensory events is fundamental to the perception of the external world

24 and adaptive behaviour. Such a representation is achieved in a spatial distribution of neuronal

25 activation which initiates communication across spatially distributed brain areas (cf. Fries, 2015).

26 Rhythmic neuronal activity or oscillations have been regarded as a key mechanism in the process

27 of neural communication in different species (Buzaki, 2006), for instance by sequencing

28 information into temporal processing windows (cf. Lopes da Silva, 1991), and by linking neural

29 assemblies through phase coherence (Tallon-Baudry, 2003; Fries, 2005, 2015). Oscillatory neural

30 activity has been associated with various brain functions (motor action, Salenius and Hari, 2003;

31 consciousness, Ward, 2003; learning and memory, Kahana, 2006; motivation and reward,

32 Knyazev, 2007; attention, Klimesch, 2012). Recent advances in the field suggest that dysfunctions

33 of the nervous system can often be traced back to disturbed network activity which lead to the

34 concept of “oscillopathies” in neurodegenerative diseases (cf. Nimmrich et al., 2015), for instance

35 revealing pathophysiological beta oscillations in Parkinson’s disease (Little and Brown, 2014),

36 gamma oscillations in Schizophrenia (Mathalon and Sohal, 2015), and both alpha and gamma

37 oscillations in Alzheimer's disease (Nimmrich et al., 2015). Notably, the identification of impaired

38 network functioning necessitates prior characterization of normal activity, beginning with the

39 sensory systems as the initial points of interaction with the external world. Such rhythmic activity

40 is currently well characterized for the visual, auditory, somatosensory, and olfactory senses in

41 various species (cf. Koepsell et al., 2010), yet insufficiently so for the gustatory system. Only

42 recently have findings provided evidence of the role of slow-wave synchronized activity in taste

43 processing in rodents (Pavao et al., 2014), whereas no studies at all have characterized the time-

44 frequency dynamics of the human gustatory system. 
46 This lack of knowledge of the frequency by which information is transmitted within the human

47 gustatory system surely does not reflect the importance of the gustatory system. On the contrary,

48 the ability to taste ensures an organism's survival by enabling the identification of nutrients and

49 avoidance of toxins via a discrimination of taste categories (often referred to as taste qualities).

50 Accordingly, taste categories have been associated with carbohydrates (sweet), electrolytes (salty),

51 acids (sour) or alkaloids (bitter). In rodents, distinct receptors on the tongue respond to chemicals

52 signifying each taste category (Chandrashekar et al., 2006) before the signal is transduced upstream

53 via the gustatory nucleus of the solitary tract in the rostral medulla, and the ventro-posterior medial

54 nucleus of the thalamus to the gustatory cortex in the insula (Carleton et al., 2010). There is

55 evidence of two competing models of taste coding: hardwired, labelled lines with specialized

56 neurons (Chen et al., 2011), and flexible, learning-dependent taste representations (Accolla et al.,

57 2007; Carleton et al., 2010), possibly through broadly tuned neurons (Stapleton et al., 2006).

58 Notwithstanding the unresolved issue of how taste categories are encoded along the peripheral

59 gustatory pathway, at the level of gustatory cortex, however, taste information can be decoded from

60 dynamic activity patterns obtained from neuronal ensembles (Jones et al., 2007) and local field

61 potentials (Pavao et al., 2014) in rodents, and large-scale EEG scalp recordings in humans (Crouzet

62 et al., 2015). The availability of taste information from large-scale recordings enables

63 investigations of the time-frequency dynamics of cortical information transfer in taste perception.

65 For our investigation, we recorded multi-channel head-surface electroencephalography (EEG) in 66 human participants while they detected salty, sweet, sour, or bitter solutions, in order to investigate 67 the neural mechanism by which the human gustatory system encodes taste information. First, we 68 investigated whether the taste-evoked electrophysiological response would selectively engage a 
69 specific frequency band, given that frequency-specific neuronal signatures have been observed in

70 other sensory systems (Koepsell et al., 2010). Since participants received four different tastants,

71 we were further able to test whether taste-specific content is represented in the frequency-specific

72 activity. Second, we hypothesized that this phenomenon would not be a mere by-product of

73 network activity, but bear functional relevance for perceptual decisions (Harmony, 2013), by

74 specifically testing whether the timing of the neural gustatory response would predict the timing at

75 which participants detect a taste. Third, because task dependency has been reported with respect to

76 taste-related behavioural responses in humans (Halpern, 1986; Bujas et al., 1989) and cortical

77 activation in rodents (Fontanini and Katz, 2009), we probed the flexibility of human gustatory

78 processing by comparing taste-evoked neural responses between the speeded detection task

79 presented here and a previously reported delayed categorization task (Crouzet et al., 2015).

MATERIALS AND METHODS

\section{Participants}

84 Sixteen healthy participants (12 women; mean age 28 \pm 5.1 years; BMI 22 \pm 3.0 ) completed the study.

85 Participants reported having no taste impairments and no history of neurological or psychiatric

86 disease. They signed informed consent prior to the start of the experiment and received monetary

87 compensation for participation. The study protocol conformed to the revised Declaration of

88 Helsinki and was approved by the ethics board of the German Psychological Society.

\section{Stimuli and taste delivery}

91 Tastants were aqueous solutions with a clear taste: $0.65 \mathrm{M}$ sodium chloride (salty;from a local

92 supermarket REWE GmbH, Köln, Germany), 0.039 M citric acid (sour; CAS 77-92-9, Sigma 
93 Aldrich, Inc., St. Louis, MO, USA), 0.44 M sucrose (sweet;from a local supermarket REWE

94 GmbH, Köln, Germany), 0.0002 M quinine monohydrate (bitter; CAS 207671-44-1, Sigma-

95 Aldrich, Inc., St. Louis, MO, USA) and 0.05 M Splenda ${ }^{\circledR}$ (sweet; Tate \& Lyle, London, UK) in

96 distilled water. Splenda trials were not included in the present analysis because they were

97 perceptually undistinguishable from sucrose. Concentrations were chosen based on previous

98 studies to be clearly perceivable and to not elicit discomfort or disgust. Taste stimuli were $210 \mu \mathrm{l}$

99 aliquots delivered at a flow rate of $233 \mu \mathrm{l} / \mathrm{s}$ during $900 \mathrm{~ms}$ as aerosol to the anterior part of the

100 slightly extended tongue with the GU002 gustometer (Burghart Messtechnik GmbH, Wedel,

101 Germany; Figure 1A). The gustometer stores taste and rinse solutions in separate bottles that each

102 supply a syringe pump with a check valve (cf. Iannilli et al., 2015). From here, solutions and

103 compressed air are transported via separate $5 \mathrm{~m}$ long Teflon tubes to a manifold outlet within a

104 spray nozzle that atomizes the liquid. The tubes run inside a hose filled with water at $38^{\circ} \mathrm{C}$ that

105 heats the stimuli which are, after atomization, delivered at near body temperature to minimize any

106 thermal sensations. The spray nozzle is positioned $1-1.5 \mathrm{~cm}$ above the slightly extended tongue so

107 that the spray covers a large area of the anterior tongue's surface. During the experiment, the

108 participant comfortably leans against a forehead rest, which stabilizes the head and holds the spray

109 nozzle in place. In this position, liquids were applied to the slightly extended tongue and not

110 swallowed but collected in a bowl underneath the chin (Figure 1B). The position was monitored

111 online via camera to ensure that the spray continuously covered the surface of the anterior tongue

112 and to monitor movements. The stimulation comprises a regular sequence of spray pulses, during

113 each of which $70 \mu \mathrm{l}$ of liquid are dispensed during $100 \mathrm{~ms}$ every $300 \mathrm{~ms}$. The timing and flow rate

114 were optimized to minimize mixing of individual spray pulses and to elicit the experience of a

115 continuous flow of liquid to the tongue. The distinct spray pulses permit to embed a taste stimulus

116 in the "flow" of control or water stimuli without tactile cue. Note that participants experience a 
117 tactile "pulsing" only for a few seconds until the lingual somatosensory system is habituated.

118 During the development of this procedure, we determined the time required for lingual habituation;

119 we measured the time to the abolishment of the lingual somatosensory steady-state response and

120 confirmed our findings with verbal reports of numbing of the tongue. The steady-state response

121 was abolished within less than $10 \mathrm{~s}$. As a result, we present water pulses for at least $10 \mathrm{~s}$ at the

122 beginning of each experimental block or experiment (see Tzieropoulos et al., 2013; Crouzet et al.,

123 2015). The time between the TTL pulses controlling the syringe plungers, which push the liquids

124 through the tubes and the spray nozzle, until the aerosol reaches the tongue's surface, was measured

125 by the supplier for the experimental setting decribed here following a previously proposed 126 conductivity measurement (Kelling, 1986). It revealed a time lag of $36 \mathrm{~ms}$ ( $\mathrm{SD}=2 \mathrm{~ms}$ ), which the 127 stimulus onset in the EEG data was corrected for.

\section{Experimental procedure}

130 Each trial (see Figure 1C) started with a central black fixation cross on gray background presented 131 for a variable amount of time (0.8-1.5 s) on a 24" thin film transistor (TFT) screen placed at a 132 distance of $45 \mathrm{~cm}$ in front of the participant. The fixation cross instructed participants to refrain 133 from any movements; it remained on screen during taste stimulation (0.9 s) until the end of the 134 response-time measurement (2.1 s). Participants were instructed to press a button on a serial 135 response box (Psychological Software Tools, Inc., Sharpsburgh, PA, USA) with the right or left 136 index finger as soon as they tasted anything (simple response-time task). Response hands were 137 switched between blocks and the starting hand was counterbalanced across participants. The 138 fixation cross was then replaced by the instruction to rate the intensity and pleasantness of the taste, 139 by moving the mouse cursor along a visual analogue scale that was labelled at the end points with 140 "no sensation" and "extremely intense" and "extremely unpleasant" to "extremely pleasant", 
141 respectively. The rating procedure lasted $8 \mathrm{~s}$ and was followed by a blank screen for $9 \mathrm{~s}$ to minimize

142 adaptation. Overall, 300 taste stimuli (60 per tastant) were presented in pseudo-random order over

143 the course of six experimental blocks. The experiment lasted approximately 120 minutes including

144 breaks between blocks and an initial training during which participants were familiarized with the

145 procedure. Participants were presented with brown noise via noise-isolating in-earphones to mask

146 any auditory cues from the spray pulses.

147

148 EEG data acquisition and pre-processing

149 Participants were seated in a sound-attenuated recording booth (Studiobox GmbH, Walzbach,

150 Germany) with the gustometer positioned outside to minimize auditory cues from the device. The

151 electroencephalogram (EEG) was recorded with an activCHamp amplifier system (Brain Products

$152 \mathrm{GmbH}$, Munich, Germany) at a sampling rate of $500 \mathrm{~Hz}$ with analogue $0.01 \mathrm{~Hz}$ highpass and 200

153 Hz lowpass filters using PyCorder (Brain Vision LLC, Morrisville, NC, USA) and with 64

$154 \mathrm{Ag} / \mathrm{AgCl}$ active electrodes placed in an elastic cap according to the extended 10-10 system. The

155 EEG data were processed offline using EEGLAB (Delorme and Makeig, 2004) in MATLAB

156 (Mathworks, Natick, MA, USA) and Autoreject (Jas et al., 2017) in Python. First, we resampled

157 the data to $200 \mathrm{~Hz}$ to improve the signal-to-noise ratio and to reduce computation times

158 (Grootswagers et al., 2017). We removed slow drifts with linear detrending and line-noise (50 Hz

159 in Germany) with a set of multi-tapers over sliding time windows implemented in the CleanLine

160 plugin. Second, we segmented the continuous data into epochs from $-1.5 \mathrm{~s}$ to $2.5 \mathrm{~s}$ relative to

161 stimulus onset and applied Autoreject for noisy channel interpolation and epoch exclusion. Less

162 than $1 \%$ of all trials were removed. Third, we computed an extended Infomax independent

163 component analysis implemented in EEGLAB (ICA; Makeig et al., 1997) to identify artefactual

164 components with manual inspection and ADJUST (Mognon et al., 2011), which uses temporal and 
165 spatial characteristics of the independent components to detect outliers. Components representing

166 common ocular, cardiac or muscular artefacts were subtracted from the data. Lastly, the data were

167 re-referenced to the average of all channels. To visualize the taste-related EEG activity, the global

168 field power (GFP), a reference-free index of overall field strength (Lehmann and Skrandies, 1980),

169 was calculated as the standard deviation over the entire electric field (i.e., all electrodes) at each

170 sampling point for each participant and for each condition separately.

171

172 Experimental design and statistical analysis

173 We employed a within-subjects design with one factor (taste category) with four levels (salty, sour,

174 bitter, sweet). All analyses were conducted within participants and are described in more detail in

175 the respective subsections below. The wavelet transformation was performed in MATLAB using

176 EEGLAB, decoding was implemented with custom scripts in R (R-Core-Team, 2014).

\section{Time-frequency decomposition}

179 To obtain a time-frequency representation of the EEG data, we computed the spectral power and

180 phase angles via continuous Morlet wavelet transforms of single trials for the frequency range from

1811 to $100 \mathrm{~Hz}$ in 40 logarithmically spaced steps). We increased the length of the wavelets linearly

182 from 1 cycle at $1 \mathrm{~Hz}$, to 15 cycles at $100 \mathrm{~Hz}$ to optimize the trade-off between temporal resolution

183 at lower frequencies and spectral precision at higher frequencies. The convolution was performed

184 non-causally (i.e. a time point is the center of the time window) in steps of $10 \mathrm{~ms}$ from -0.2 to 1.5

185 s. The result of the convolution is a complex number of which the magnitude represents the spectral

186 power and the angle the phase. For visualization, we quantified the degree of event-related phase

187 synchronisation across trials with the inter-trial coherence (ITC; cf. Busch et al., 2009), which takes

188 values between 0 (no synchronisation) and 1 (perfect synchronisation). 


\section{Taste discrimination analysis}

191 To search for taste information in the time-frequency spectrum, we performed a multivariate 192 pattern analysis (MVPA, cf. Kriegeskorte, 2011) at every time and frequency step, using the log193 transformed spectral power and phase angles in radians. The MVPA was implemented with a L2194 regularized linear support vector machine (SVM; Fan et al., 2008) within subjects in a stratified

195 leave-one-trial-out cross-validation (CV; i.e. on every iteration, a trial of each taste is left out) for 196 optimal model estimation. The multi-taste classification problem was solved via pairwise binary

197 classifiers with the final performance as the average over the six one-versus-one comparisons (cf.

198 Hand and Till, 2001). Thus, the decoder would see the instantaneous topographical distribution of 199 the spectral power or phase angles of a large subset of trials and learn the taste-specific 200 systematicity in their patterns. The availability of such taste-specific information was tested by 201 generalizing these patterns to the taste-evoked responses of the left-out trials.

203 We optimized the regularization constant $C$ in incremental steps of negative powers of $10\left(10^{-4: 0}\right)$

204 in a nested CV. In this tuning protocol, the training set was iterated in an inner 10-fold stratified 205 CV to search for the $\mathrm{C}$ which achieved optimal performance across time points on the inner subset 206 of the data. The classifier was then trained on the full training set with the best value for $\mathrm{C}$ of the 207 inner tuning set and tested for its performance on the left-out trials which were neither part of 208 training nor tuning. We evaluated the classifier performance with the area under the receiver 209 operating characteristic curve (AUC), which provides a balanced accuracy metric (50\% 210 performance corresponds to chance). For the assessment of statistical significance $(p<.05)$ and

211 adjustment for multiple null hypothesis testing, we performed cluster-based permutation analyses 212 on the AUC scores (Maris and Oostenveld, 2007). 


\section{Task dependency of taste coding}

215 After identification of the frequency band that contained taste information, we performed an

216 MVPA which incorporated both power and phase characteristics to compare taste information

217 coding between the task reported in the current study (hereafter, speeded taste detection) with a

218 previously reported task, during which participants were to taste the stimuli and to discriminate

219 tastes only after presentation (hereafter, passive or delayed taste discrimination; Crouzet et al.,

220 2015). For this, we isolated the signal via zero-phase Hamming-windowed sinc finite impulse

221 response (FIR) filter ( $-6 \mathrm{~dB}$ cutoff, maximum passband deviation of $0.2 \%$, stopband attenuation

222 of $-53 \mathrm{~dB}$ ). Epochs were resampled to $100 \mathrm{~Hz}$ to match the temporal resolution of the wavelet

223 analysis, and filtering was performed on epochs from $-1.2 \mathrm{~s}$ up to $2.5 \mathrm{~s}$ to minimize aliasing before

224 reducing the epochs to the -0.2 to $1.5 \mathrm{~s}$ interval of interest. The decoding procedure was performed

225 as described above (see Taste discrimination analysis). Statistical significance of above-chance

226 performance was assessed via one-sided binomial tests and adjusted to a minimum of $100 \mathrm{~ms}$ of $p$ -

227 values $<.05$. Differences between the decoding performance scores for the two tasks were

228 compared via two-sided Wilcoxon rank sum tests and adjusted to a minimum of two consecutive 229 time points.

\section{Controlling for the influence of motor activation}

232 To ensure that the classifiers were not using information related to the motor response in order to

233 discriminate between the tastes, we ran a control analysis in which we repeated the multiclass

234 MVPA with a careful separation of response sides. Here, we trained the classifiers to discriminate

235 between tastes by means of the electrophysiological patterns of trials with a left button press, and

236 tested their performance on patterns of trials with a right button press, and vice versa (out of sample 
237 classification). If the classification performance was comparable to the performance found for taste

238 decoding this would demonstrate that the classifier is independent of motor activation and that it

239 indeed used taste-specific information.

241 Correspondence between neural and behavioural responses

242 To test whether the neural taste signal predicts taste detection times, we estimated signal onsets for

243 single trials. We performed the MVPA within a taste category to discriminate a trial against its

244 water baseline (average of $200 \mathrm{~ms}$ prestimulus). This analysis is ideal for estimating the

245 classification performance independently of the contrast to the other tastes, and the decoding task

246 closely resembled the taste-detection task that participants performed. More specifically, a

247 classifier was to decide at every time point of a trial whether the neural signal shows a taste-

248 response or still reflects the water rinse, essentially mirroring the decision participants performed

249 between water rinse and the detection of a taste in that very same trial in which the signal was

250 obtained.

251 We applied the same CV and tuning protocol as for the previous analyses. Trials without responses

252 or with responses faster than 100 ms were excluded (3.5\% of trials). To determine the time at which

253 taste patterns emerged at the level of single trials, we used a strict adjustment procedure. To account

254 for cases in which the correct label may be assigned by chance, we estimated for each correct

255 decision a decoder's confidence through Platt scaling (Platt, 1999) and computed the lower $5^{\text {th }}$

256 percentile of this confidence estimate for each C parameter (regularization shrinks confidence).

257 The onset within a trial (i.e. the emergence of a pattern which successfully distinguishes a taste

258 from water) was determined as the earliest point before a psychomotor response at which the

259 decoder's decision was correct for at least $100 \mathrm{~ms}$ and with a confidence level above the (C-

260 dependent) $5^{\text {th }}$ percentile threshold. Trials without such a streak of correct classification were 
261 removed from the analysis (17.4\% of trials). We opted for this conservative procedure because any

262 'decoding onset’ beyond the button press would be an indication of a poor signal-to-noise ratio or

263 could be related to the motor response itself.

264 We computed hierarchical linear mixed regression models to examine the link between the 265 estimated onsets as the predictor of response times. We defined the intercepts as random effects at

266 the second level (participants), and the intercepts and slopes as random effects at the third level

267 (tastes). To examine whether the emerging patterns could be linked to other taste features besides 268 taste identity, we also computed analogous models with participants' rating scores of taste intensity 269 and pleasantness as the dependent variable. We report the fixed effects with their standard errors 270 and estimated $p$-values, using $\left(\chi^{2}\right.$-distributed) likelihood ratio-tests against the restricted (intercept271 only) null models.

\section{Orofacial electromyography}

274 To test whether taste-specific orofacial behavior may have influenced the present data, we recorded 275 surface electromyography (EMG) in a healthy female participant during tasting. To validate the 276 placement of the EMG electrodes, we recorded $60 \mathrm{~s}$ EMG to three types of movement relevant to 277 tasting: tongue movements, and the emotional facial expressions related to disgust (pouting) and 278 pleasure (smile). The movement-related EMG also served as a benchmark for signal strength 279 because we expected little to no EMG activity during tasting because participants kept a slightly 280 opened jaw and an extended tongue during the experiment (see Figure 1B), which inhibits oromotor 281 behaviour such as tongue, lip or jaw movements and innate craniofacial responses of the lower 282 facial area, which are known to vary between non-sweet tastes (Rosenstein and Oster, 1988). To 283 increase the saliency of the taste stimuli and with that the likelihood to evoke a facial and/or oro284 motor reaction, we used a higher flow rate compared to the EEG study and presented the tastants 
as single pulses (not embedded in a stream of water) using a computer-controlled gustometer based

286 on neMESYS syringe pumps (Cetoni, Korbußen, Germany), which delivers liquids at higher flow

287 rates with otherwise comparable stimulus properties to the EEG study (Andersen et al., 2018).

288 Tastants were $0.675 \mathrm{ml}$ vaporized aliquots with a flow rate of $0.75 \mathrm{ml} / \mathrm{s}$ that were applied to the 289 anterior third of the tongue and washed off with a water rinse of $2 \mathrm{ml}$ at a flow rate of $0.5 \mathrm{ml} / \mathrm{s}$.

290 EMG was recorded at $1 \mathrm{kHz}$ with $15 \mathrm{~mm} 3$ adhesive $\mathrm{Ag} / \mathrm{AgCl}$ electrodes (BlueSensor N, Ambu 291 A/S, Ballerup, Denmark) and snap-on wires connected to the PowerLab 26T amplifier 292 (ADInstruments, Oxford, United Kingdom) using the LabChart Pro 8.1.10 software 293 (ADInstruments, Oxford, United Kingdom). During recording, data were filtered with a $0.5 \mathrm{~Hz}$ 294 highpass and $2 \mathrm{kHz}$ lowpass filters. We recorded from four different muscles: the left masseter, 295 which controls mastication and jaw movements, the left zygomaticus major, which controls the 296 smile, the right mentalis, which controls the chin and contributes to pouting, and the right 297 infrahyoid muscles, which play a role in swallowing. The sides of the face were chosen to allow 298 convenient placement of the electrodes and to avoid tangling of cables. For each muscle, two 299 electrodes were spaced approx. $1 \mathrm{~cm}$ apart and the ground electrode was placed on the dorsum of 300 the right hand.

301 The participant leaned against a forehead rest and extended the tongue with a slightly opened jaw 302 while fixating a central crosshair presented on a gray screen. Tastants were presented in blocks 303 with 20 stimuli. Movements were recorded at random times between taste blocks. Each trial started 304 with a central fixation cross presented for 2-3 s on a gray background before a taste stimulus was 305 presented for $900 \mathrm{~ms}$, while the fixation cross remained on the screen. After a pause of 2 s, a water 306 rinse was delivered for 4 s. A gray screen was displayed during the rinse until the next trial. The 307 ISI was 20-21 s. Each of four tastants was presented 10 times in pseudo-random order. Because 308 the amplifier allowed simultaneous recording of only two muscles, we conducted the experiment 
309 twice, with muscles and tasks counterbalanced. We found no obvious EMG during tasting (in

310 contrast to movement) suggesting that the reported EEG effects are not confounded by taste-

311 specific orofacial behavior (see Inline Supplementary Figure 1).

313 RESULTS

\section{Taste information encoded by delta activity}

315 All four tastes evoked a taste-related electrophysiological response that is similar to previous 316 reports (Tzieropoulos et al., 2013; Crouzet et al., 2015; Fig. 1D). To investigate the encoding of

317 taste information at a specific frequency, we used SVM classifiers at each time and frequency step 318 to decode the four tastes using either the spectral power (Fig. 2A) or the phase angles (visualized 319 as ITC in Fig. 2C). We found significant clusters of increased classification performance in the

320 lower part of the frequency spectrum. The classifiers decoded taste-specific information from the 321 activation patterns of the spectral power in a major cluster largely contained within the 1 to $4 \mathrm{~Hz}$ 322 band (30 to $1160 \mathrm{~ms}$; $\mathrm{AUC}_{\max }=56.4 \% \pm 1.2, p<.0001$, at $1.4 \mathrm{~Hz}$ and $360 \mathrm{~ms}$; Fig 2B), which 323 extended into the 8-12 Hz range between 480 and $860 \mathrm{~ms}$. Similarly, decoding of the phase angles 324 revealed a major cluster contained within the frequency spectrum below $8 \mathrm{~Hz}$ (10 to $1260 \mathrm{~ms}$; $325 \mathrm{AUC}_{\max }=64.3 \% \pm 2.0, p<.0001$, at $2.9 \mathrm{~Hz}$ and $300 \mathrm{~ms}$; Fig 2D). This finding suggests that taste 326 identity is encoded mainly by power and phase in the delta-frequency range (1-4 Hz), and therefore, 327 the subsequent analysis focused solely on this frequency.

329 To optimize temporal precision, we decoded taste identity information from the electrical 330 recordings after band-pass zero-phase FIR filtering $(0.5-4.5 \mathrm{~Hz} \pm 1$; order: 330$)$ to isolate delta 331 activity from other frequencies. We identified the earliest point of significant above-chance 332 classification performance at $130 \mathrm{~ms}(\mathrm{AUC}=53.0 \% \pm 1.4, p=.005)$; it lasted until the end of the 
333 epoch $\left(\mathrm{AUC}_{\max }=63.7 \% \pm 1.7, p<.0001\right.$, at $450 \mathrm{~ms}$; Fig. 2E). Moreover, in contrast to the unfiltered

334 recordings, the delta-encoded signal yielded higher classification performance throughout the 335 epoch, suggesting an overall improved signal-to-noise ratio (AUC maxdiff $=5.3 \%$ at $460 \mathrm{~ms}, p=.007$;

336 cf. Fig. 2E). Notably, taste patterns appeared to emerge earlier during this speeded taste-detection 337 task than in a previously reported study, in which participants gave delayed taste-categorization 338 responses (Crouzet et al., 2015). To test this, we applied the multi-class decoding analysis reported

339 here using the FIR filtered electrophysiological recordings obtained during the delayed tasting task 340 and compared the findings (Fig. 2F). We found that classification performance reached significance

$34140 \mathrm{~ms}$ later in the delayed task, compared to the speeded one (at $170 \mathrm{~ms}$, AUC $=52.6 \% \pm 1.4, p$ $342=.022)$. The classification performance of the speeded task was significantly higher than the 343 delayed task during the periods from 140 to $170 \mathrm{~ms}$ and from 320 to $360 \mathrm{~ms}$ (all $p<.0001$;

$344 \mathrm{AUC}_{\text {maxdiff }}=4.7 \%$ at $160 \mathrm{~ms}$ ). In contrast, the classification performance of the delayed task was 345 significantly higher in later periods from 740 to $860 \mathrm{~ms}$ and from 950 to $990 \mathrm{~ms}$ (all $p<.0001$;

$346 \quad \mathrm{AUC}_{\text {maxdiff }}=4.7 \%$ at $790 \mathrm{~ms}$ ). Together, these findings indicate that the taste response pattern is 347 susceptible to task demands.

349 To exclude the possibility that the classifiers were using motor-related activation to solve the multi350 taste discrimination, we reiterated the procedure by carefully separating the response sides, so that 351 classifiers were trained on patterns from trials with right-sided responses and tested on patterns of 352 trials with left-sided responses, and vice versa. We found highly similar performance curves for 353 classifiers trained on right- and left-sided responses with above-chance classification starting at $354130 \mathrm{~ms}$ for most of the time range for both response sides (training left, testing right: AUC $_{\text {onset }}=$ 355 53.6\% $\pm 1.0, p=.022, \mathrm{AUC}_{\max }=61.0 \% \pm 1.3$ at $520 \mathrm{~ms}$; training right, testing left: $\mathrm{AUC}_{\text {onset }}=$ $35653.6 \% \pm 1.1, p=.026$; $\mathrm{AUC}_{\max }=63.0 \% \pm 1.7$ at $400 \mathrm{~ms}$; Figure 2G). Importantly, the performance 
357 curves for response-side and taste decoding were highly similar as well (compare Figures 2E and

358 2G). The interchangeability of the response side topographies during classification confirms that

359 the taste discriminability within the delta spectrum was independent of motor activation.

\section{The emergence of taste-evoked delta activity is predictive of taste detection responses}

362 The above findings indicate delta activation as an electrophysiological signature of taste-

363 information coding. To test whether there was a systematic link between these patterns and taste

364 detection times, we computed multi-level linear mixed models between the onset of delta-encoded

365 taste information and response times. We used SVM classifiers to discriminate each trial from its

366 baseline period water rinse, which closely matched the taste-detection task that participants

367 performed in a given trial (Fig. 3A). We found a highly significant positive relationship between

368 the onsets of taste-pattern decoding and taste detection response times $\left(\beta=.32 \pm .03, \chi^{2}(1, N=2990)=\right.$

$36916.87, p<.0001$; Fig. 3B) for each of the four tastes: salty $\left(M_{\mathrm{on}}=185 \mathrm{~ms}, M_{\mathrm{RT}}=643 \mathrm{~ms}, \beta=.35\right)$,

$370 \operatorname{sour}\left(M_{\mathrm{on}}=196 \mathrm{~ms}, M_{\mathrm{RT}}=680 \mathrm{~ms}, \beta=.34\right)$, sweet $\left(M_{\mathrm{on}}=242 \mathrm{~ms}, M_{\mathrm{RT}}=845 \mathrm{~ms}, \beta=.30\right)$, and

371 bitter $\left(M_{\mathrm{on}}=244 \mathrm{~ms}, M_{\mathrm{RT}}=798 \mathrm{~ms}, \beta=.31\right)$. This finding indicates a functional relevance of this

372 activation pattern for taste detection behavior, such that faster taste-pattern emergence can be

373 linked to faster taste-detection times.

375 To determine whether the observed delta activity was encoding taste identity rather than taste 376 intensity or valence, we computed analogous mixed linear models using the intensity and 377 pleasantness ratings as dependent variables. We observed no significant relationship between the

378 onsets of taste-pattern decoding (quantified in ms) and intensity ratings $\left(\beta=-.004 \pm .002, \chi^{2}(1, N=2984)\right.$

$379=2.33, p=.127 ; M_{\text {salty }}=74, M_{\text {sour }}=79, M_{\text {sweet }}=51, M_{\text {bitter }}=66$; see Inline Supplementary Figure

$3801 \mathrm{~A})$, and pleasantness ratings $\left(\beta=.001 \pm .002, \chi_{(1, N=2985)}^{2}=0.15, p=.699 ; M_{\text {salty }}=27, M_{\text {sour }}=27\right.$, 
$M_{\text {sweet }}=65, M_{\text {bitter }}=26$; see Inline Supplementary Figure 1B). Together, these results suggest that

382 delta activity encodes information of taste identity rather than other taste features.

383

384

385

386

387

388

389

390

391

392

393

394

395

396

397

398

399

400

401

402

403

404

405

\section{DISCUSSION}

The fundamental role of coherent rhythmic activity in neuronal communication has been well established for perception and cognition across sensory systems and species (von Stein et al., 2000;

Engel et al., 2001; Varela et al., 2001; Fries, 2005; Buzaki, 2006). Here, we identified for the first time delta activity $(1-4 \mathrm{~Hz})$ as a distinct electrophysiological signature of gustatory processing in the human brain. This finding adds to recent evidence that highlights the relevance of slow cortical rhythms in the coding of taste-specific information in the rodent gustatory cortex ( $<1.5$ and 4-5 Hz; Pavao et al., 2014) and odor-specific content in the human olfactory cortex (4-8 Hz; Jiang et al., 2017).

Primate taste processing involves neural computations in a widespread network, including the insula (primary gustatory area) and the orbitofrontal cortex (secondary gustatory area) (Pritchard and Di Lorenzo, 2015), which further link to subcortical structures regulating reward and feeding behavior (Katz and Sadacca, 2011), as well as somatosensory and visceral areas (Katz et al., 2002). Given that the length of an oscillatory cycle determines the range and timescale of cortical integration, such that slower oscillations bridge longer distances and accomplish more complex computations (Engel et al., 2010; Harmony, 2013), our findings of delta-encoded taste information may indicate the mechanism by which the human brain communicates within such a spatially distributed and highly interconnected system. Delta oscillations have been found to originate in the primate insula (Mesulam and Mufson, 1982), and they play a role in food-related arousal such as craving and reward (cf. Knyazev, 2007, 2012). We supplement this knowledge with evidence of a 
novel role of delta activity in coding taste information in the human brain. Here, the slow-wave

407 activity subsides with the offset of stimulation. Given the successful and improved classification

408 of the taste quality information from the spectral signatures after attenuation of non-delta

409 frequencies, we interpret this finding in accordance with the literature on slow rhythmic activity,

410 which has been suggested to subserve the sequencing of a sensory experience into discrete

411 processing entities (Colgin, 2013; Wilson et al., 2015). Accordingly, each processing cycle

412 coordinates through its phase the activation of cell populations (phase coherence) that represent

413 specific experiential aspects, whereas the cycle sequence links the individual segments to maintain

414 the integrity of the experience. Thus, information is encoded in one region, and by means of a

415 specific phase and cycle, segregated information bits become accessible to distant brain areas by

416 'reading' this oscillatory activity. Our findings suggest that delta activity contributes the candidate

417 frequency with which such distinct information packages are transmitted from the same taste

418 episode across the long spatial trajectory of the taste network. This interpretation will benefit from

419 additional electrocorticographical data and modelling.

420 In line with the notion that phase coherence coordinates neuronal activation, we found that the

421 phase encoded more information than its amplitude. More than a means of coordinating clearly

422 defined bits of information, in sensory cortices, the functional role of slow oscillations has been

423 proposed as an internal reference frame which stabilizes the encoding of natural stimuli - analogous

424 to a metronome which links and integrates the spike patterns of single neurons to a coherent code

425 - thereby fostering perceptual robustness (Panzeri et al., 2014). This notion is especially intriguing

426 for the chemical senses, for which sensory representations have to be formed in environments with

427 a high degree of (temporal) uncertainty (cf. Jiang et al., 2017). Among other factors, the gustatory

428 system encounters noise as sensory inputs originate from different spatial locations within the oral

429 cavity over an extended period of mastication and swallowing with no precisely timed stimulus. 
430 Indeed, the action potentials of (chemo-)sensory neurons are much more precisely timed than the

431 chemical stimulation they respond to. When these action potentials are summed over long time 432 windows, sensory information is at risk to be degraded or lost. Accordingly, an oscillatory 433 reference frame may act as a pacemaker, which helps decode the incoming, segregated information 434 from finely timed spike patterns of possibly distant areas (Panzeri et al., 2014). Thus, the gustatory 435 system, like the olfactory, would benefit particularly from slow rhythmic activity as a means of 436 attaining perceptual robustness.

438 To determine whether delta-encoded taste information is in fact used for perceptual decisions, we 439 correlated the onset of delta activity with the behavioral response time in each trial. This onset 440 predicted when participants detected a given taste, such that a more rapid pattern-emergence 441 corresponded to faster response behavior. Importantly, we excluded the possibility of decoding 442 performance being confounded by motor signals through careful cross-response side validation. 443 This apparent neural-behavioral link aligns well with the aforementioned notion that slow 444 oscillations are not just a by-product of network processing, but have functional significance for 445 the perceptual integration and temporal alignment of sensory inputs through coherence (e.g. via 446 delay-based synchrony detection). Moreover - although the units by which neurons encode 447 information are precisely timed spike patterns within a range of a few milliseconds - the 448 behaviorally relevant information may very well extend over hundreds of milliseconds. Therefore, 449 slow rhythmic patterns can encode information that is not available in local spike counts (Kayser 450 et al., 2012), and thus enable other brain structures to extract relevant sensory information which 451 informs decisions or concomitant perceptual states (Wilson et al., 2015). Indeed, the present data 452 provide evidence of such a link between the timing of delta activity and subsequent behavior. 453 
454 To test whether the timing of the earliest sensory taste-response is independent of behavioral

455 demands, we compared the decoding performance between two different tasks: a fast paced, 456 speeded taste-detection task (reported here) and a slow paced, delayed taste-categorization task

457 (Crouzet et al., 2015). We found earlier and stronger delta-encoded taste discriminability in the 458 speeded and more sustained taste discriminability in the delayed task, suggesting that neural taste

459 responses are contingent upon behavioral goals. Admittedly, the present data do not allow to 460 conclusively identify the aspect of the task that drives that difference. Task-dependent latency 461 differences in event-related potentials have been observed previously for other modalities. Here we 462 show that task-dependent latency shifts reflect actual shifts in the availability of taste category 463 information. The observed flexibility in taste-information coding accords well with the observation 464 that gustatory cortical activity varies greatly with expectation and focus of attention in rodents 465 (Fontanini and Katz, 2009). In fact, it has been shown that the influence of cueing is maximal in 466 the early portion of gustatory cortex responses by increasing the amount of selectively firing 467 neurons, sharpening their tuning, and reducing variability (Samuelsen et al., 2012). Even though 468 both (speeded and delayed) experimental tasks employed cueing, it is plausible that the task itself 469 introduces a cue-like state change to the responsiveness of the gustatory cortex. Consequently, the 470 degree and speed at which the taste system categorizes stimuli is likely to be tuned to situational 471 demands, thereby enabling adaptive behaviour.

472

473 In summary, we present evidence of activation in the delta-frequency range as an 474 electrophysiological signature of taste processing in humans. We show that taste-specific content 475 can be discerned from these signatures, which predict perceptual decisions and align flexibly with 476 task requirements. 


\section{Conflict of Interest}

The authors declare no competing financial interests.

\section{Acknowledgements}

This work was supported by NutriAct - Competence Cluster Nutrition Research Berlin-Potsdam funded by the Federal Ministry of Education and Research (FKZ: 01EA1408A-G) granted to KO. 


\section{References}

Accolla R, Bathellier B, Petersen CC, Carleton A (2007) Differential spatial representation of taste modalities in the rat gustatory cortex. The Journal of neuroscience : the official journal of the Society for Neuroscience 27:1396-1404.

Andersen CA, Alfine L, Ohla K, Höchenberger R (2018) A new gustometer: Template for the construction of a portable and modular stimulator for taste and lingual touch. In. Behavioral Research Methods (manuscript in revision).

Bujas Z, Szabo S, Ajdukovic D, Mayer D (1989) Individual gustatory reaction times to various groups of chemicals that provoke basic taste qualities. Perception \& psychophysics 45:385390.

Busch NA, Dubois J, VanRullen R (2009) The phase of ongoing EEG oscillations predicts visual perception. The Journal of neuroscience : the official journal of the Society for Neuroscience 29:7869-7876.

Buzaki G (2006) Rhythms of The Brain: Oxford University Press.

Carleton A, Accolla R, Simon SA (2010) Coding in the mammalian gustatory system. Trends in neurosciences 33:326-334.

Chandrashekar J, Hoon MA, Ryba NJP, Zuker CS (2006) The receptors and cells for mammalian taste. Nature 444:288-294.

Chen X, Gabitto M, Peng Y, Ryba NJP, Zuker CS (2011) A gustotopic map of taste qualities in the mammalian brain. Science (New York, NY) 333:1262-1266.

Colgin LL (2013) Mechanisms and functions of theta rhythms. Annu Rev Neurosci 36:295-312.

Crouzet SM, Busch NA, Ohla K (2015) Taste quality decoding parallels taste sensations. Current Biology 25(7):890-896.

Delorme A, Makeig S (2004) EEGLAB: an open source toolbox for analysis of single-trial EEG dynamics including independent component analysis. Journal of neuroscience methods 134:9-21.

Engel AK, Fries P, Singer W (2001) Dynamic predictions: oscillations and synchrony in top-down processing. Nature reviews Neuroscience 2:704-716.

Engel AK, Friston K, Kelso JAS, König P, Kovács I, MacDonald III A, Miller EK, Phillips WA, Silverstein SM, Tallon-Baudry C, Triesch J, Uhlhaas P (2010) Coordination in Behavior and Cognition. In: Dynamic Coordination in the Brain: From Neurons to Mind (von der Malsburg C, Phillips WA, Singer W, eds), pp 267-300: MIT Press.

Fan R, Chang K, Hsieh C (2008) LIBLINEAR: A library for large linear classification. The Journal of Machine Learning Research 9:1871-1874.

Fontanini A, Katz DB (2009) Behavioral modulation of gustatory cortical activity. Annals of the New York Academy of Sciences 1170:403-406.

Fries P (2005) A mechanism for cognitive dynamics: neuronal communication through neuronal coherence. Trends in cognitive sciences 9:474-480.

Fries P (2015) Rhythms for Cognition: Communication through Coherence. Neuron 88:220-235.

Grootswagers T, Wardle SG, Carlson TA (2017) Decoding Dynamic Brain Patterns from Evoked Responses: A Tutorial on Multivariate Pattern Analysis Applied to Time Series Neuroimaging Data. Journal of cognitive neuroscience 29:677-697.

Halpern BP (1986) Constraints imposed on taste physiology by human taste reaction time data. Neuroscience and biobehavioral reviews 10:135-151.

Hand DJ, Till RJ (2001) A simple generalisation of the area under the ROC curve for multiple class classification problems. Machine learning 45:171-186. 
Harmony $\mathrm{T}$ (2013) The functional significance of delta oscillations in cognitive processing. Frontiers in integrative neuroscience 7:83.

Herrmann CS, Grigutsch M, Busch NA (2005) EEG oscillations and wavelet analysis. In: Eventrelated Potentials: A Methods Handbook (Handy TC, ed), pp 229-259. Cambridge, MA: MIT Press.

Iannilli E, Beger M, Furer R, Hummel T (2015) A gustatory stimulator. Journal of neuroscience methods 255:12-16.

Jas M, Engemann DA, Bekhti Y, Raimondo F, Gramfort A (2017) Autoreject: Automated artifact rejection for MEG and EEG data. In, 12 Jul 2017 Edition, pp 1-24. arXiv.

Jiang H, Schuele S, Rosenow J, Zelano C, Parvizi J, Tao JX, Wu S, Gottfried JA (2017) Theta Oscillations Rapidly Convey Odor-Specific Content in Human Piriform Cortex. Neuron 94:207-219 e204.

Jones LM, Fontanini A, Sadacca BF, Miller P, Katz DB (2007) Natural stimuli evoke dynamic sequences of states in sensory cortical ensembles. Proceedings of the National Academy of Sciences of the United States of America 104:18772-18777.

Kahana MJ (2006) The cognitive correlates of human brain oscillations. The Journal of neuroscience : the official journal of the Society for Neuroscience 26:1669-1672.

Katz DB, Sadacca BF (2011) Taste. In: Neurobiology of Sensation and Reward (Gottfried JA, ed). Boca Raton, FL, USA: CRC Press/Taylor \& Francis.

Katz DB, Nicolelis MA, Simon SA (2002) Gustatory processing is dynamic and distributed. Current opinion in neurobiology 12:448-454.

Kayser C, Ince RA, Panzeri S (2012) Analysis of slow (theta) oscillations as a potential temporal reference frame for information coding in sensory cortices. PLoS computational biology 8:e1002717.

Kelling ST, Halpern, B. P. (1986) The physical characteristics of of open flow and closed flow taste delivery apparatus. Chemical senses 11:89-104.

Klimesch W (2012) alpha-band oscillations, attention, and controlled access to stored information. Trends in cognitive sciences 16:606-617.

Knyazev GG (2007) Motivation, emotion, and their inhibitory control mirrored in brain oscillations. Neuroscience and biobehavioral reviews 31:377-395.

Knyazev GG (2012) EEG delta oscillations as a correlate of basic homeostatic and motivational processes. Neuroscience and biobehavioral reviews 36:677-695.

Koepsell K, Wang X, Hirsch JA, Sommer FT (2010) Exploring the function of neural oscillations in early sensory systems. Frontiers in neuroscience 4:53.

Kriegeskorte N (2011) Pattern-information analysis: from stimulus decoding to computationalmodel testing. NeuroImage 56:411-421.

Lehmann D, Skrandies W (1980) Reference-free identification of components of checkerboardevoked multichannel potential fields. Electroencephalography and clinical neurophysiology 48:609-621.

Little S, Brown P (2014) The functional role of beta oscillations in Parkinson's disease. Parkinsonism Relat Disord 20 Suppl 1:S44-48.

Lopes da Silva F (1991) Neural mechanisms underlying brain waves: from neural membranes to networks. Electroencephalography and clinical neurophysiology 79:81-93.

Makeig S, Jung TP, Bell AJ, Ghahremani D, Sejnowski TJ (1997) Blind separation of auditory event-related brain responses into independent components. Proceedings of the National Academy of Sciences of the United States of America 94:10979-10984. 
Maris E, Oostenveld R (2007) Nonparametric statistical testing of EEG- and MEG-data. Journal of neuroscience methods 164:177-190.

Mathalon DH, Sohal VS (2015) Neural Oscillations and Synchrony in Brain Dysfunction and Neuropsychiatric Disorders: It's About Time. JAMA Psychiatry 72:840-844.

Mesulam MM, Mufson EJ (1982) Insula of the old world monkey. III: Efferent cortical output and comments on function. The Journal of comparative neurology 212:38-52.

Mognon A, Jovicich J, Bruzzone L, Buiatti M (2011) ADJUST: An automatic EEG artifact detector based on the joint use of spatial and temporal features. Psychophysiology 48:229-240.

Nimmrich V, Draguhn A, Axmacher N (2015) Neuronal Network Oscillations in Neurodegenerative Diseases. Neuromolecular Med 17:270-284.

Panzeri S, Ince RA, Diamond ME, Kayser C (2014) Reading spike timing without a clock: intrinsic decoding of spike trains. Philosophical transactions of the Royal Society of London Series B, Biological sciences 369:20120467.

Pavao R, Piette CE, Lopes-dos-Santos V, Katz DB, Tort ABL (2014) Local field potentials in the gustatory cortex carry taste information. The Journal of neuroscience : the official journal of the Society for Neuroscience 34:8778-8787.

Platt J (1999) Probabilistic outputs for support vector machines and comparisons to regularized likelihood methods. Advances in large margin classifiers 10:61-74.

Pritchard TC, Di Lorenzo PM (2015) Central Taste Anatomy and Physiology of Rodents and Primates. In: Handbook of Olfaction and Gustation (Doty RL, ed), pp 701-726. Hoboken, NJ, USA: John Wiley \& Sons, Inc.

R-Core-Team (2014) R: A Language and Environment for Statistical Computing. In. Vienna, Austria: R Foundation for Statistical Computing.

Rosenstein D, Oster H (1988) Differential facial responses to four basic tastes in newborns. Child Dev 59:1555-1568.

Salenius S, Hari R (2003) Synchronous cortical oscillatory activity during motor action. Current opinion in neurobiology 13:678-684.

Samuelsen CL, Gardner MP, Fontanini A (2012) Effects of cue-triggered expectation on cortical processing of taste. Neuron 74:410-422.

Stapleton JR, Lavine ML, Wolpert RL, Nicolelis MA, Simon SA (2006) Rapid taste responses in the gustatory cortex during licking. The Journal of neuroscience : the official journal of the Society for Neuroscience 26:4126-4138.

Tallon-Baudry C (2003) Oscillatory synchrony and human visual cognition. Journal of physiology, Paris 97:355-363.

Tzieropoulos H, Rytz A, Hudry J, le Coutre J (2013) Dietary fat induces sustained reward response in the human brain without primary taste cortex discrimination. Frontiers in human neuroscience 7:36.

Varela F, Lachaux JP, Rodriguez E, Martinerie J (2001) The brainweb: phase synchronization and large-scale integration. Nature reviews Neuroscience 2:229-239.

von Stein A, Chiang C, Konig P (2000) Top-down processing mediated by interareal synchronization. Proceedings of the National Academy of Sciences of the United States of America 97:14748-14753.

Ward LM (2003) Synchronous neural oscillations and cognitive processes. Trends in cognitive sciences 7:553-559.

Wilson MA, Varela C, Remondes M (2015) Phase organization of network computations. Current opinion in neurobiology 31:250-253. 
618 FIGURES AND FIGURE LEGENDS

619

\section{Figure 1. Experimental setup.}

621 A: The GU002 gustometer (Burghart Medizintechnik, Wedel, Germany) delivers liquids via 622 separate lines and at constant temperature. B: Vaporized tastants and rinse are applied to the 623 extended, anterior tongue. Tastants are not ingested but collected in a reservoir underneath the 624 participant's chin. C: Schematic depiction of a trial including the display, taste stimulation, and 625 response. Atomized tastants (black) were embedded in a sequence of water (grey). Motor responses 626 were recorded between taste onset and rating prompt. D: Grand-averaged global field power (GFP) 627 for each of four tastes. Stimulus onset is at $0 \mathrm{~ms}$. 

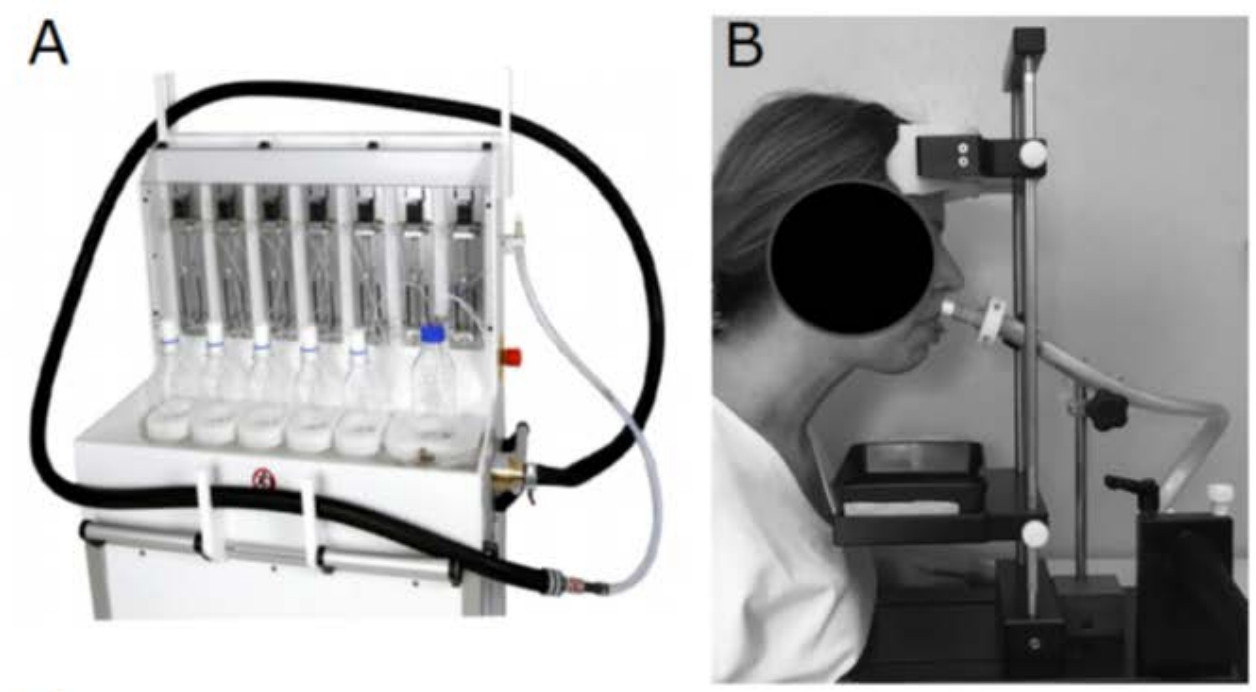

C

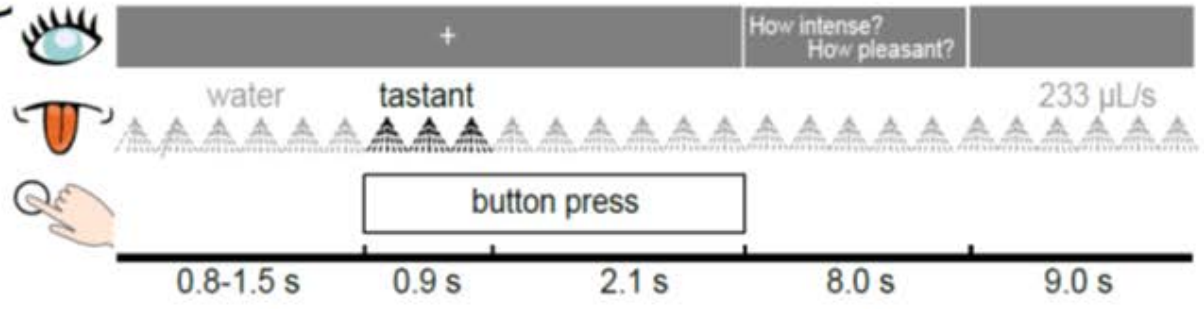

D

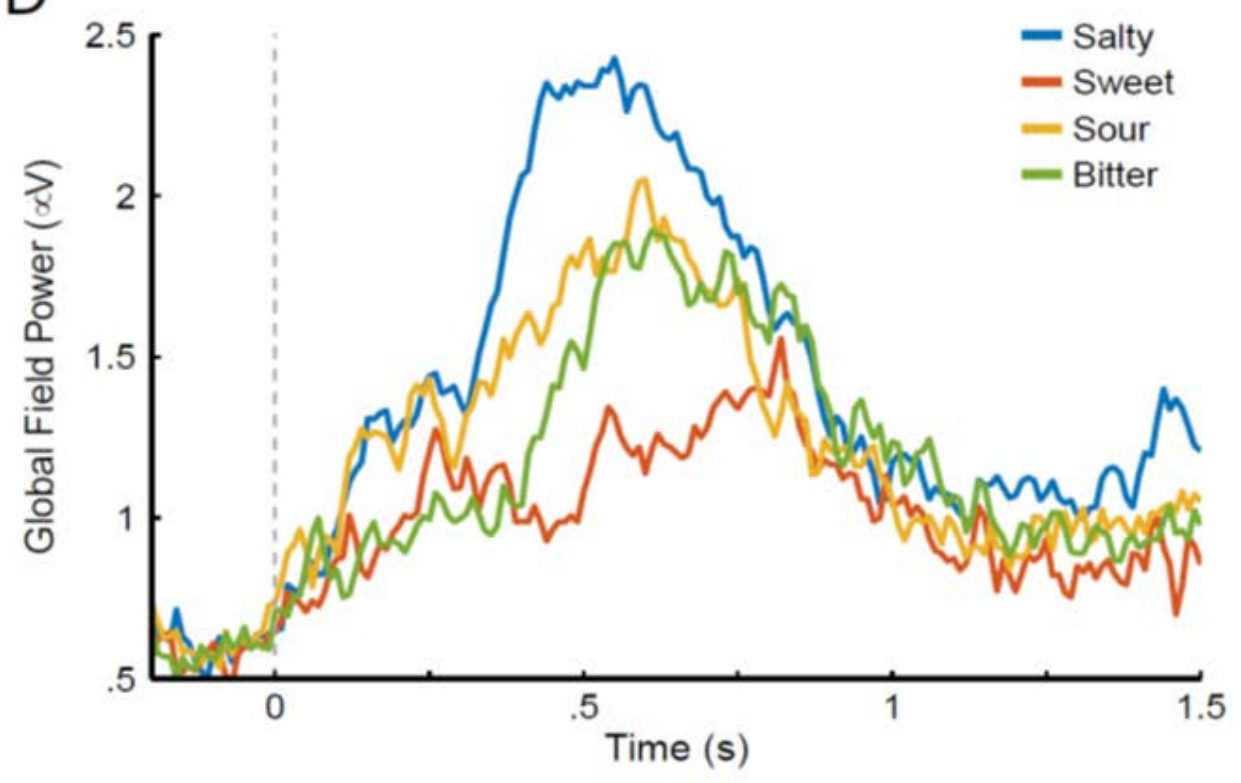

628 
629 Figure 2. Taste information is encoded by delta oscillations.

630 A) Spectral power estimates obtained via continuous wavelet transformation of the EEG recordings 631 for frequencies from 1 to $100 \mathrm{~Hz}$ and averaged across 64 electrodes and 16 participants. The power 632 represents the amplitude of the signal. Increases (warm colours) and decreases (cold colours) are 633 relative to the baseline (for visualization). Vertical dashed line: stimulus onset; horizontal dotted 634 lines: transitions between established frequency bands (1-4 Hz: delta, 4-8 Hz: theta, 8-12 Hz: alpha, 635 12-30 Hz: beta, 30-100 Hz: gamma; cf. Herrmann et al., 2005). B) SVM classifiers were trained at 636 each time point and frequency step to decode the four tastes given the (non-normalized) spectral 637 power in the 64-electrode space. Performance is displayed as the AUC (50\% corresponds to the 638 chance level) averaged across participants (black contour lines indicate cluster-corrected 639 significance). C) Phase-locking value or inter-trial coherence (ITC) is calculated from phase 640 estimates obtained via continuous wavelet transformation for frequencies from 1 to $100 \mathrm{~Hz}$. The 641 ITC expresses the extent of phase synchronization across trials (remainder as in A). D) SVM 642 classifiers were trained at each time point and frequency step to decode the four tastes given the 643 phases in radians in the 64-electrode space (remainder as in B). E) Comparison of taste response644 pattern decoding between the broad-band and delta signal (1-4 Hz). SVM classifiers were trained 645 at each time point to decode the four tastes, given the unfiltered (black) or FIR filtered (blue) 646 electrophysiological recordings from 64 electrodes (solid lines: mean AUC across trials and 647 participants; surrounding shaded regions \pm 1 SEM; colour-coded horizontal lines above the $\mathrm{x}$-axis 648 indicate above-chance performance; horizontal dotted line: theoretical chance level of 50\%). F) 649 Comparison between the speeded task of the current study and a delayed task (Crouzet et al., 2015). 650 Colour-coded stars at the top indicate significantly different classification performance between 651 tasks; colour-coded dashed vertical lines indicate task-specific starting times of significant multi652 taste discrimination (remainder as in E). G) Motor-control task. Response mappings were separated 653 for the training and test set, i.e. the decoder was trained on trials with a left button press, and tested 654 on trials with a right button press (and vice versa). Successful classification demonstrates that 655 motor-related activity is irrelevant to the multi-taste discrimination (remainder as in E). 


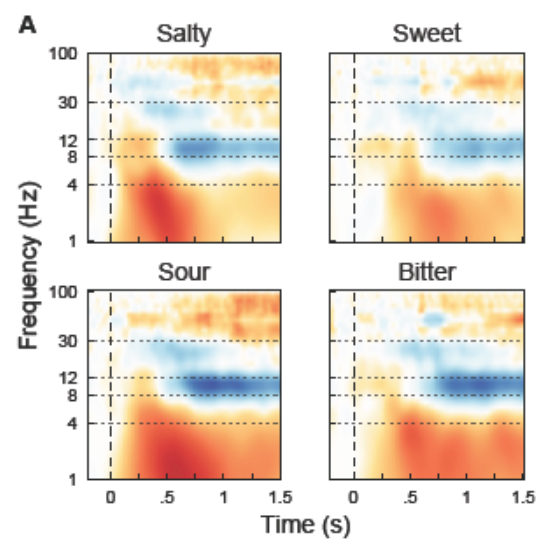

A

$$
\text { Power }(\Delta \mathrm{dB})
$$

C

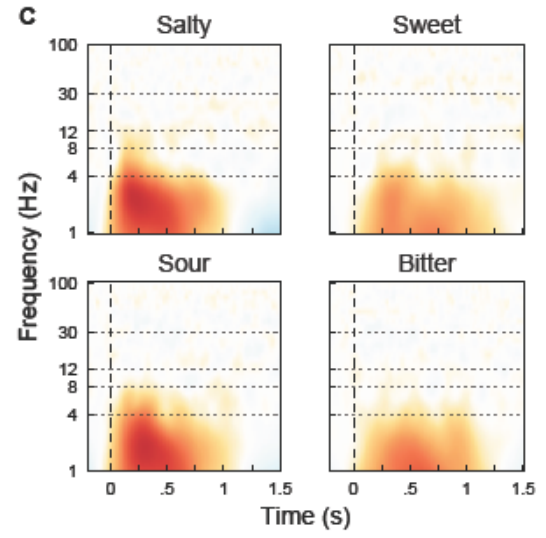

Phase Locking $(\Delta \Phi)$

657

658

659

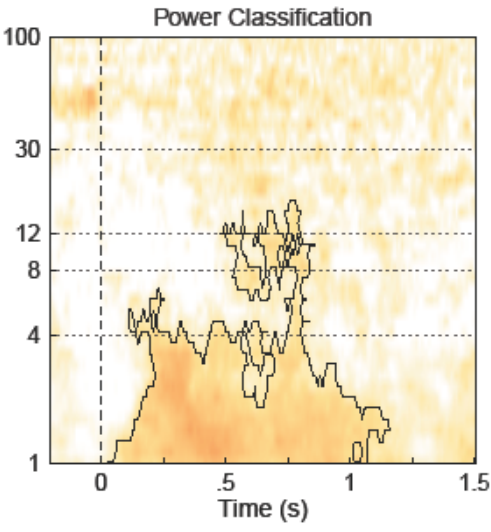

AUC (\%)

$\begin{array}{llll}50 & 55 & 60 & 65\end{array}$

D

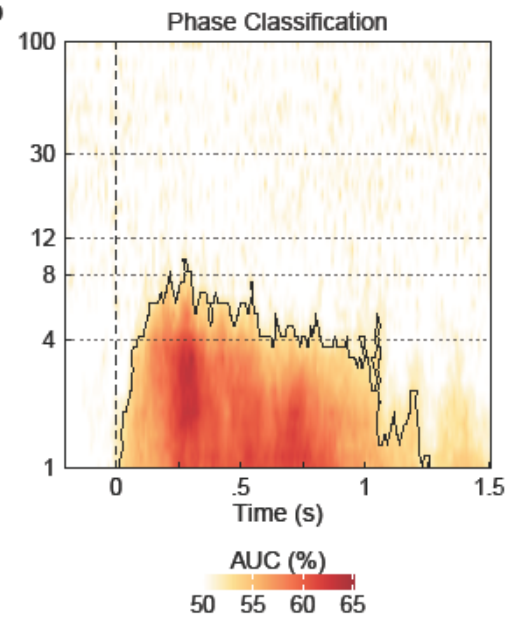

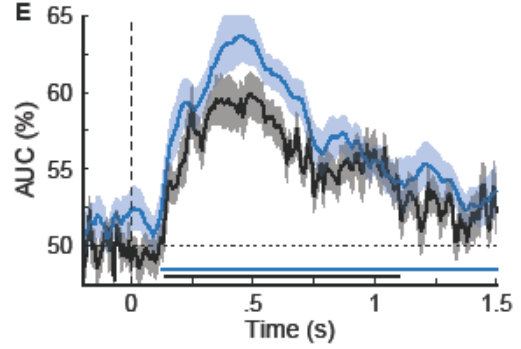

Spectrum - Broad - Delta

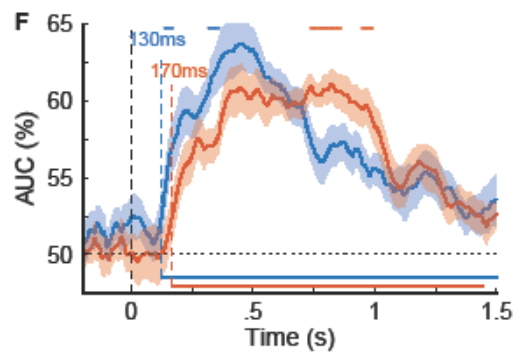

Task - Passive - Active

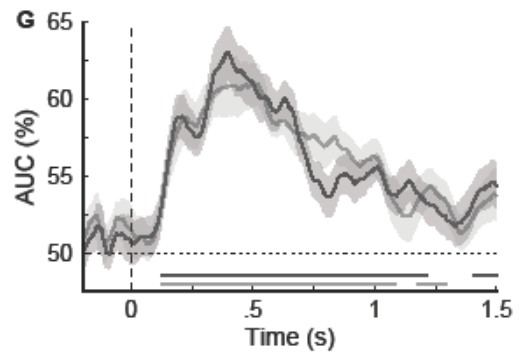

Response Side $=$ Left - Right 
660 Figure 3. The emergence of the delta-encoded electrophysiological taste signature is 661 predictive of taste responses. A) SVM classifiers were trained within participants at each time 662 point on the patterns of N-1 trials, in order to decode taste and water in the left-out trial (each trial 663 was matched with its water baseline average). This classification task closely corresponds to the 664 taste-detection task participants performed (press as soon as you taste something). Colour-coded 665 solid lines: mean AUC across participants, surrounding shaded regions \pm 1 SEM; colour-coded 666 horizontal lines above the x-axis indicate periods of above-chance performance per participant; 667 colour-coded dashed vertical lines: average onset time; black dashed vertical lines: average 668 response time; grey vertical dashed line: stimulus onset; black horizontal dotted line: theoretical 669 chance level of 50\%. B) Correlation plots between the onsets of pattern decoding (determined as 670 the earliest point at which a trial has been classified successfully for at least $100 \mathrm{~ms}$ ) and taste671 detection response times. Color-coded solid lines indicate the taste-specific influence of decoding 672 onset from the multi-level linear mixed regression fits, grey dashed vertical lines at $1.5 \mathrm{~s}$ indicate 673 the end of the EEG epochs. 
A
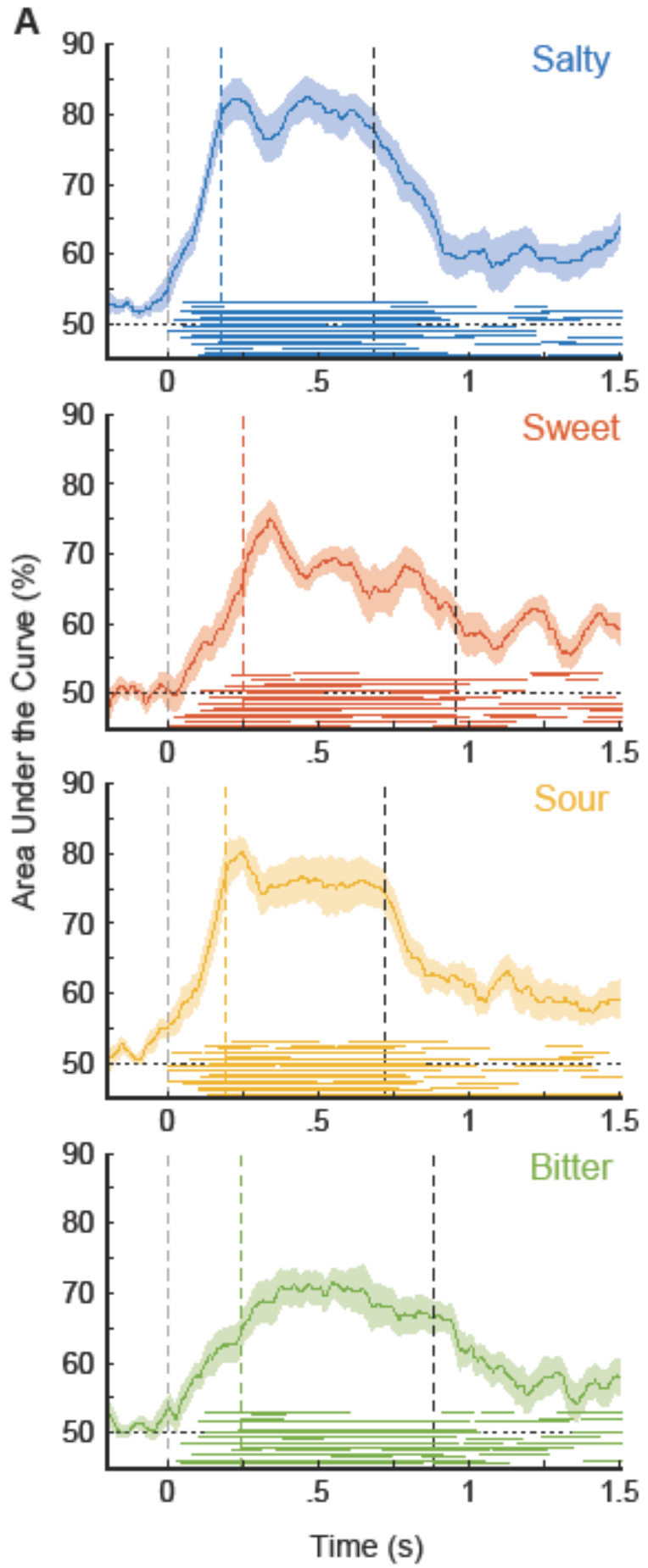

B
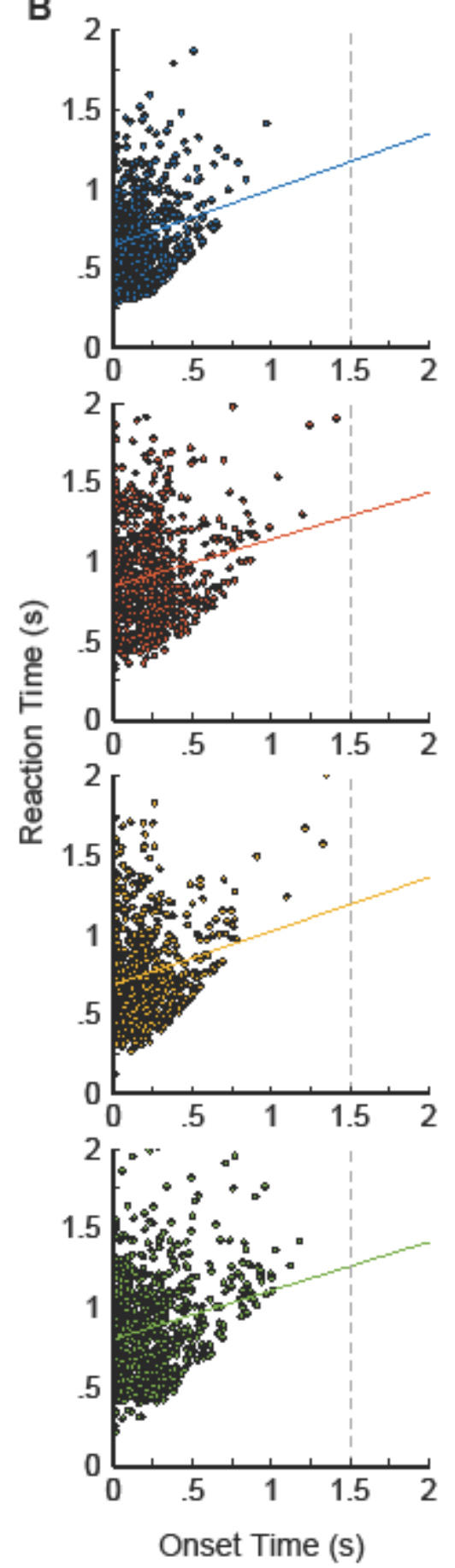

675

676 